\title{
Does Marital Status Influence Levels of Anxiety and Depression Before Palliative Radiotherapy?
}

\author{
CARSTEN NIEDER ${ }^{1,2}$ and THOMAS A. KÄMPE ${ }^{1}$ \\ ${ }^{1}$ Department of Oncology and Palliative Medicine, Nordland Hospital Trust, Bod $\phi$, Norway; \\ ${ }^{2}$ Department of Clinical Medicine, Faculty of Health Sciences, \\ UiT - The Artic University of Norway, Troms $\phi$, Norway
}

\begin{abstract}
Background/Aim: To evaluate whether or not single patients report increased levels of anxiety and depression, compared to married or partnered patients scheduled to receive palliative radiotherapy. In principle, different levels of social support might cause such disparities. Patients and Methods: Retrospective comparison of two groups of patients (28\% single, overall 100 patients), who scored their symptoms before palliative radiotherapy with the Edmonton symptom assessment system (ESAS). Results: The two groups differed significantly with regard to irradiated target sites (more brain irradiation in the married/partnered group), receipt of systemic therapy, which was more common in the married/partnered group, and mean age (single patients were older). Mean anxiety and depression scores were not significantly different between the two groups. Survival was similar, too (median 6 months, $p=0.77)$. Conclusion: Similar ESAS scores of anxiety and depression were observed in the two groups (single vs. married/partnered patients).
\end{abstract}

Marital support plays an important role regarding different aspects of cancer care and might be associated with better treatment compliance and intensity (1-3). Lack of marital and other types of social support might result in negative consequences, e.g., disparities regarding medication compliance, adherence to supportive measures and lower patient-reported quality of life $(2,3)$. Many cancer patients are treated with palliative aim and receive multi-modal approaches, which often include radiotherapy (4-7). In

This article is freely accessible online.

Correspondence to: Carsten Nieder, Department of Oncology and Palliative Medicine, Nordland Hospital Trust, 8092 Bodø, Norway. Tel: +47 75578449, Fax: +47 75534975, e-mail: carsten.nieder@nlsh.no

Key Words: Palliative radiotherapy, Edmonton symptom assessment system, anxiety, depression, marital status. previous studies, patients scheduled for radiotherapy have reported heightened levels of anxiety and/or depression (8, 9). We hypothesized that single patients scheduled for palliative radiotherapy might report increased levels of anxiety and depression, compared to married or partnered patients with presumed better social support. In order to test this hypothesis, a secondary analysis of a single institution study was performed (10), which employed previously collected questionnaires (Edmonton symptom assessment system, ESAS). This short, one-sheet questionnaire has been used by different radiotherapy departments and addresses major symptoms and wellbeing on a numeric scale of 0-10 (highest symptom severity 10), including anxiety and depression $(9,11,12)$.

\section{Patients and Methods}

The study included 100 of 102 patients at an academic teaching hospital who received palliative radiotherapy during the time period 2013-2015 (10). Two patients had to be excluded because their marital status was unknown. The ESAS tool was administered by a registered oncology nurse immediately before oncologist consultation and imaging for treatment planning, i.e. approximately one week before palliative radiotherapy. All medical records, except for in-patient psychiatric care, were available in the hospital's electronic patient record system. Statistical analysis was performed with IBM SPSS Statistics 24 . We analyzed two different subgroups, single patients and those who were married or partnered. We employed the chi-square test (when appropriate Fisher exact probability test or $t$-test). A $p$-value $\leq 0.05$ was considered statistically significant. Two-tailed tests were performed. Actuarial survival from start of radiotherapy was analyzed with the KaplanMeier method and log-rank test. Ethical approval was not required for this secondary analysis in accordance with the national and institutional guidelines.

\section{Results}

Twenty-eight patients (28\%) were single, 65 married and 7 partnered. For different age groups up to 79 years, the majority of patients was married or partnered. However, 
Table I. Baseline characteristics before palliative radiotherapy.

\begin{tabular}{|c|c|c|c|c|c|}
\hline Characteristics & $\begin{array}{c}\text { No } \\
\text { Single }\end{array}$ & $\%$ & $\begin{array}{c}\text { No } \\
\text { M/P }\end{array}$ & $\%$ & $p$-Value \\
\hline \multicolumn{6}{|l|}{ Gender } \\
\hline Male & 20 & 71 & 54 & 75 & \\
\hline Female & 8 & 29 & 18 & 25 & 0.80 \\
\hline \multicolumn{6}{|l|}{ Primary tumor site } \\
\hline Prostate & 11 & 39 & 20 & 28 & \\
\hline Breast & 2 & 7 & 10 & 14 & \\
\hline Lung & 7 & 25 & 20 & 28 & \\
\hline Colorectal & 0 & 0 & 5 & 7 & \\
\hline Bladder & 3 & 11 & 2 & 3 & \\
\hline Malignant melanoma & 1 & 4 & 3 & 4 & \\
\hline Others & 4 & 14 & 12 & 17 & $0.63^{*}$ \\
\hline \multicolumn{6}{|l|}{ Disease stage } \\
\hline Distant metastases & 23 & 82 & 67 & 93 & \\
\hline No distant metastases & 5 & 18 & 5 & 7 & 0.14 \\
\hline \multicolumn{6}{|l|}{ RT target type } \\
\hline Bone metastases & 16 & 57 & 41 & 57 & \\
\hline Brain metastases & 0 & 0 & 11 & 15 & \\
\hline Lymph node metastases & 1 & 4 & 4 & 6 & \\
\hline Lung & 5 & 18 & 4 & 6 & \\
\hline Prostate or bladder & 4 & 14 & 5 & 7 & \\
\hline Others & 2 & 7 & 7 & 10 & $0.04 * *$ \\
\hline \multicolumn{6}{|l|}{ RT fractionation } \\
\hline$>10$ fractions & 5 & 18 & 16 & 22 & \\
\hline 10 fractions & 10 & 36 & 31 & 43 & \\
\hline 5-9 fractions & 8 & 29 & 20 & 28 & \\
\hline$<5$ fractions & 5 & 18 & 5 & 7 & 0.42 \\
\hline \multicolumn{6}{|l|}{ Systemic cancer treatment } \\
\hline No & 9 & 32 & 10 & 14 & \\
\hline Before RT & 19 & 68 & 62 & 86 & 0.05 \\
\hline Mean time from first cancer diagnosis to RT (months) & 50 & & 55 & & 0.68 \\
\hline Mean ECOG PS & 2.0 & & 1.7 & & 0.19 \\
\hline Mean age & 74 & & 70 & & 0.05 \\
\hline
\end{tabular}

M/P: Married or partner; RT: radiotherapy; ECOG PS: Eastern Cooperative Oncology Group performance status. *collapsed into 4 categories (prostate/breast/lung/other). **Collapsed into 4 categories (bone/brain/lung/other).

among the oldest patients $(\mathrm{n}=25$ above the age of 79 years $)$ the majority was single (60\%). Further patient characteristics are shown in Table I. Married or partnered patients more often were irradiated to brain target volumes and less often to lungs, prostate or bladder $(p=0.04)$. They were also more likely to have received systemic therapy before radiotherapy $(p=0.05)$. Furthermore, they were significantly younger $(p=0.05)$.

Mean anxiety and depression scores were not significantly different between the two groups, as illustrated in Table II. Significant differences were seen for one ESAS item only, namely overall wellbeing $(p=0.015)$. Single patients reported better scores, together with trends towards less pain, fatigue and nausea, and better sleep. Actuarial survival was not significantly different, with a median of 6 months in both groups $(p=0.77)$.

\section{Discussion}

The background of this study was concern about inappropriate social support in single patients, that could result in heightened levels of anxiety and/or depression. According to ESAS-derived data, similar scores of anxiety and depression were observed in the two groups (single vs. married/partnered patients). Unexpectedly, single patients reported significantly better overall wellbeing and trends towards better sleep, less pain, fatigue and nausea. The fact that single patients were significantly older did thus not result in inferior ESAS scores. Probably, differences in disease extent existed between both groups, as indicated by imbalances in systemic treatment utilization and types of irradiated target volumes. Although this information was not available in our database, it is tempting to speculate that 
Table II. Association between ESAS scores and marital status.

\begin{tabular}{lccc}
\hline Parameter & $\begin{array}{c}\text { Single } \\
\text { Mean, SD, range }\end{array}$ & $\begin{array}{c}\text { Married/partner } \\
\text { Mean, SD, range }\end{array}$ & $p$-Value \\
\hline Anxiety & $2.79,2.64,0-9$ & $2.62,3.15,0-10$ & 0.81 \\
Depression & $1.71,2.05,0-6$ & $2.35,2.99,0-10$ & 0.31 \\
Pain (not moving) & $2.25,2.27,0-7$ & $3.07,2.73,0-9$ & 0.16 \\
Pain (moving) & $3.96,2.55,0-8$ & $4.58,3.41,0-10$ & 0.39 \\
Fatigue & $3.79,3.01,0-10$ & $4.72,2.85,0-10$ & 0.15 \\
Nausea & $0.82,1.61,0-6$ & $1.35,2.00,0-8$ & 0.22 \\
Appetite & $3.46,2.99,0-10$ & $3.90,3.39,0-10$ & 0.55 \\
Constipation & $2.70,3.22,0-10$ & $2.40,3.02,0-10$ & 0.67 \\
Dry mouth & $3.00,2.69,0-10$ & $3.00,2.86,0-9$ & 1.0 \\
Dyspnea & $2.64,2.50,0-8$ & $2.69,2.92,0-10$ & 0.94 \\
Sleep & $1.96,2.53,0-8$ & $2.89,2.83,0-10$ & 0.14 \\
Overall wellbeing & $2.68,2.14,0-6$ & $4.04,2.56,0-10$ & 0.015 \\
\hline
\end{tabular}

many of the elderly single patients were widowed. The exact level of social support was not captured, because no specific questionnaires for this endpoint were used. In theory, some single patients might have a sufficient network of friends and relatives. Among the limitations of this study one has also to discuss the cohort size, which has implications for the statistical power. Furthermore, it remains unknown how many patients utilized medications that are prescribed for anxiety or depression when they scored their symptoms.

An Italian study of 544 oncological inpatients employed different tools, i.e. the Hospital Anxiety and Depression Scale and the Needs Evaluation Questionnaire (13). In this cohort, $27.4 \%$ and $20.8 \%$ of the enrolled patients were probable cases, respectively, for anxiety and depression. When possible, cases were included and the percentages raised to $52.5 \%$ and $39.3 \%$, respectively. Few differences in anxiety and depression according to socio-demographic and clinical variables were demonstrated. A different crosssectional study that used a different anxiety and depression scale was carried out in 150 outpatients with cancer in Pakistan (14). Marital status had no significant influence on the presence of anxiety and depression. In a Canadian study of 326 patients with metastatic gastrointestinal or lung cancer multivariate analyses indicated that referral for specialized psychosocial oncology care was associated with unmarried status (15). A study from Australia included 266 palliative care patients with cancer who were assessed with the Hospital Anxiety and Depression Scale (16). Overall, 45.8\% of patients were possibly depressed and $22.7 \%$ probably depressed; $36.9 \%$ were possibly anxious and $19.8 \%$ probably anxious. Marital status predicted probable anxiety, but not depression. Taken together, the studies did not provide strong evidence for clinically relevant associations between marital status and anxiety/depression. However, they were performed in different cultural and oncological settings, and with heterogeneous methodology. As discussed by several groups, ESAS is a relatively crude screening tool $(9,11)$. Other approaches are available; however, the time aspect is of concern when large numbers of patients are to be evaluated, especially in busy oncology departments $(8,17)$. Overall, anxiety and depression are common in palliatively treated cancer patients and must not be overlooked.

\section{Conclusion}

The facts that similar ESAS scores of anxiety and depression were observed and that prognosis was comparable suggest that marital status, as defined in the present study, was of limited relevance.

\section{References}

1 Puts MT, Papoutsis A, Springall E and Tourangeau AE: A systematic review of unmet needs of newly diagnosed older cancer patients undergoing active cancer treatment. Support Care Cancer 20: 1377-1394, 2012.

2 Picard L: Social issues: marital support. Cancer Treat Res 151: 441-450, 2009.

3 Kiserud CE, Dahl AA, Loge JH and Fosså SD: Cancer survivorship in adults. Recent Results Cancer Res 197: 103-120, 2014.

4 Janssen S, Bolm L, Käsmann L, Bartscht T and Rades D: Palliative radiation therapy for spinal cord compression from metastatic soft tissue sarcoma. In Vivo 30: 529-531, 2016.

5 Rades D, Sehmisch L, Bajrovic A, Janssen S and Schild SE: Comparison of $20 \times 2$ Gy and $12 \times 3$ Gy for whole-brain irradiation of multiple brain metastases from malignant melanoma. In Vivo 30: 917-919, 2016.

6 Nieder C, Mannsåker B, Dalhaug A, Pawinski A and Haukland E: Palliative radiotherapy in cancer patients with increased serum C-reactive protein level. In Vivo 30: 581-586, 2016.

7 Nieder C, Dalhaug A, Pawinski A, Mannsåker B and Haukland E: Survival after palliative radiotherapy in patients with breast cancer and bone-only metastases. In Vivo 30: 879-883, 2016.

8 Mackenzie LJ, Carey M, Sanson-Fisher R, D'Este C and Yoong SL: A cross-sectional study of radiation oncology outpatients' concern about, preferences for, and perceived barriers to discussing anxiety and depression. Psychooncology 24: 13921397, 2015.

9 Körner P, Ehrmann K, Hartmannsgruber J, Metz M, Steigerwald $\mathrm{S}$, Flentje $\mathrm{M}$ and van Oorschot B: Patient-reported symptoms during radiotherapy: Clinically relevant symptom burden in patients treated with palliative and curative intent. Strahlenther Onkol 193: 570-577, 2017.

10 Nieder C, Kämpe TA and Engljähringer K: Does patient-reported dyspnea reflect thoracic disease characteristics in patients with incurable cancer? Anticancer Res 38: 901-904, 2018.

11 Salvo N, Zeng L, Zhang L, Leung M, Khan L, Presutti R, Nguyen J, Holden L, Culleton S and Chow E: Frequency of reporting and predictive factors for anxiety and depression in patients with advanced cancer. Clin Oncol (R Coll Radiol) 24: 139-148, 2012. 
12 Khan L, Kwong J, Nguyen J, Chow E, Zhang L, Culleton S, Zeng L, Jon F, Tsao M, Barnes E, Danjoux C, Sahgal A and Holden L: Comparing baseline symptom severity and demographics over two time periods in an outpatient palliative radiotherapy clinic. Support Care Cancer 20: 549-555, 2012.

13 Annunziata MA, Muzzatti B, Bidoli E and Veronesi A: Emotional distress and needs in Italian cancer patients: prevalence and associations with socio-demographic and clinical factors. Tumori 98: 119-125, 2012.

14 Jadoon NA, Munir W, Shahzad MA and Choudhry ZS: Assessment of depression and anxiety in adult cancer outpatients: a cross-sectional study. BMC Cancer 10: 594, 2010.

15 Ellis J, Lin J, Walsh A, Lo C, Shepherd FA, Moore M, Li M, Gagliese L, Zimmermann C and Rodin G: Predictors of referral for specialized psychosocial oncology care in patients with metastatic cancer: the contributions of age, distress, and marital status. J Clin Oncol 27: 699-705, 2009.
16 O'Connor M, White K, Kristjanson LJ, Cousins K and Wilkes $\mathrm{L}$ : The prevalence of anxiety and depression in palliative care patients with cancer in Western Australia and New South Wales. Med J Aust 193: S44-47, 2010.

17 Rhondali W, Perceau E, Berthiller J, Saltel P, Trillet-Lenoir V, Tredan O, Coulon JP, Bruera E and Filbet M: Frequency of depression among oncology outpatients and association with other symptoms. Support Care Cancer 20: 2795-2802, 2012.

Received December 13, 2017

Revised January 3, 2018

Accepted January 4, 2018 